\title{
Unexpected Death of a Child with Complex Febrile Seizures - Pathophysiology Similar to Sudden Unexpected Death in Epilepsy?
}

\begin{abstract}
Brian J. Dlouhy ${ }^{1,2 *}$, Michael A. Ciliberto ${ }^{3}$, Christina L. Cifra ${ }^{3}$, Patricia A. Kirby ${ }^{4}$, Devin L. Shrock ${ }^{4}$, Marcus Nashelsky ${ }^{4 * t}$ and George B. Richerson ${ }^{2,5 * t}$

'Department of Neurosurgery, University of lowa Hospitals and Clinics, lowa City, IA, USA, ${ }^{2}$ Pappajohn Biomedical Institute, University of lowa Carver College of Medicine, lowa City, IA, USA, ${ }^{3}$ Department of Pediatrics, University of lowa Hospitals and Clinics, lowa City, IA, USA, ${ }^{4}$ Department of Pathology, University of lowa Hospitals and Clinics, lowa City, IA, USA, ${ }^{5}$ Department of Neurology, University of lowa Hospitals and Clinics, lowa City, IA, USA
\end{abstract}

\section{OPEN ACCESS}

Edited by:

Christopher Michael DeGiorgio,

University of California

Los Angeles, USA

Reviewed by: Steven Neal Roper,

University of Florida, USA Antonio Gambardella, Magna Græcia University, Italy

*Correspondence: Brian J. Dlouhy

brian-dlouhy@uiowa.edu; Marcus Nashelsky marcus-nashelsky@uiowa.edu; George B. Richerson george-richerson@uiowa.edu

tThese authors have contributed equally to this work.

Specialty section: This article was submitted to Epilepsy,

a section of the journal

Frontiers in Neurology

Received: 24 September 2016 Accepted: 13 January 2017 Published: 01 February 2017

Citation:

Dlouhy BJ, Ciliberto MA, Cifra CL, Kirby PA, Shrock DL, Nashelsky M and Richerson GB (2017) Unexpected Death of a Child with Complex Febrile SeizuresPathophysiology Similar to Sudden Unexpected Death in Epilepsy?

Front. Neurol. 8:21.

doi: 10.3389/fneur.2017.00021
Febrile seizures are usually considered relatively benign. Although some cases of sudden unexplained death in childhood have a history of febrile seizures, no documented case of febrile seizure-induced death has been reported. Here, we describe a child with complex febrile seizures who died suddenly and unexpectedly after a suspected seizure while in bed at night during the beginning phases of sleep. She was resuscitated and pronounced brain dead 2 days later at our regional medical center. Autopsy revealed multiorgan effects of hypoperfusion and did not reveal an underlying (precipitating) disease, injury, or toxicological cause of death. Although a seizure was not witnessed, it was suspected as the underlying cause of death based on the medical examiner and forensic pathologist (author Marcus Nashelsky) investigation, the post-resuscitation clinical findings, and multiple aspects of the clinical history. The child had a history of complex febrile seizures that had previously caused apnea and oxygen desaturation. She had two febrile seizures earlier on the same day of the fatal event. Interestingly, her mother also experienced a febrile seizure as a child, which led to respiratory arrest requiring cardiorespiratory resuscitation. This case suggests that in a child with complex febrile seizures, a seizure can induce death in a manner that is consistent with the majority of cases of sudden unexpected death in epilepsy (SUDEP). Further work is needed to better understand how and why certain individuals, with a history of epilepsy or not, die suddenly and unexpectedly from seizures. This will only occur through better understanding of the pathophysiologic mechanisms underlying epileptic and febrile seizures and death from seizures including SUDEP.

Keywords: febrile seizures, epilepsy, sudden unexplained death in childhood, sudden unexpected death in epilepsy, sudden infant death syndrome, sudden death in the young, sudden unexpected infant death

\section{INTRODUCTION}

Febrile seizures are usually considered relatively benign, and no reported cases of febrile seizureinduced death have been documented $(1,2)$. By contrast, sudden unexpected death in epilepsy (SUDEP) is a well-established phenomenon but excludes patients without a diagnosis of epilepsy (3). However, in susceptible individuals, the pathophysiologic mechanisms underlying SUDEP (4) 
could potentially be induced by a first unprovoked seizure, or even by a provoked seizure (5). Increasing evidence suggests that some cases of sudden unexplained death in childhood (SUDC) are associated with a history of febrile seizures $(6,7)$.

Here, we describe a case of a child with complex febrile seizures who died suddenly and unexpectedly after a suspected seizure while in bed at night, with her face partially turned and covered, and during the beginning phases of sleep. We propose that this death may have resulted from the same pathophysiologic mechanisms as those believed to occur in the majority of SUDEP cases as detailed in MORTEMUS (8), outlined in multiple reviews (4, 5, 9) and discussed in recent studies examining the pathophysiology surrounding SUDEP (10)—a generalized tonic-clonic seizure (GTCS) leads to ictal and postictal respiratory dysfunction and hypoxemia, which is exacerbated by being facedown in bed, during sleep, and which ultimately leads to bradycardia and asystole.

\section{METHODS}

All inpatient and outpatient records were reviewed, and patient demographics, clinical history, clinical presentation, radiographic findings, medical examiner autopsy findings, and neuropathology were recorded and analyzed. Written and informed consent was obtained.

\section{CASE REPORT}

A 21-month-old girl with normal healthy development and without significant past medical history initially presented twice on the same day with two witnessed complex febrile GTCSs 12 months prior to her death. One seizure led to loss of breathing and oxygen desaturation with cyanosis of the lips and cyanosis and mottling of the skin. The child was febrile to $39.4^{\circ} \mathrm{C}$ in our emergency department following the second seizure. She was postictal, sleepy, and ataxic, with an otherwise normal examination and was admitted for observation. Her hospital course was unremarkable, and she was discharged the following day.

One month later, she had a simple febrile seizure in the setting of croup and, 7 months later, had two more GTCSs in the setting of another upper respiratory illness. One of these seizures occurred at night while in bed with her mother, who awakened to the GTCS. The child again became cyanotic with the seizure, requiring stimulation by her mother, and ultimately began breathing again in the postictal period. In all of these episodes, she quickly returned to baseline after the seizures and the febrile illnesses resolved without complication.

She continued to develop normally and, at 33 months of age, she had witnessed another GTCS. At that time, she was seen in the emergency department, where she was postictal and sleepy with a temperature of $38.2^{\circ} \mathrm{C}$. She was otherwise normal on neurological examination. A few hours later, she had witnessed another GTCS with postictal drowsiness. She recovered but did not return to her baseline level of activity that day. That night, she was placed in her parents' bed to sleep between her mother and her sister. When last seen alive by her father, she was on her left side facing her sister. Approximately $30 \mathrm{~min}$ later, her father saw that she was still on her left side closely adjacent to her sister, who was also on her left side. Her face was close to a pillow and her sister's back. The father found that she was unresponsive, pulseless, and apneic.

Her mother, a certified registered nurse anesthetist, and paramedics performed cardiorespiratory resuscitation. There was eventual return of spontaneous circulation after approximately $30 \mathrm{~min}$ after being found unresponsive. She was transported to our hospital. Neurological examination revealed no brainstem reflexes and no movement of the extremities. A CT scan of the brain showed diffuse swelling of the cerebral hemispheres, consistent with hypoperfusion brain injury. An electroencephalogram showed severe generalized voltage suppression without clear cerebral activity. An echocardiogram revealed adequate cardiac function with a normal heart rate and blood pressure. A chest radiograph showed airspace changes in the upper lobes of the lungs. Clinical evaluation revealed no evidence of traumatic injury. She was pronounced brain dead 2 days after admission. Her parents consented to organ donation, which was followed by autopsy performed by a medical examiner (author Marcus Nashelsky).

The cause of death was determined by the medical examiner and forensic pathologist (author Marcus Nashelsky) to be "global hypoxic/ischemic encephalopathy due to prolonged, resuscitated cardiopulmonary arrest due to complications of recurrent complex febrile seizures or epilepsy." At autopsy, no toxicological or structural underlying cause of death was identified. Neuropathological examination of the brain revealed global cerebral edema and marked softening of the brain with central, uncal, and tonsillar herniation-expected findings days after an anoxic brain injury. No masses were identified. Microscopy revealed diffuse hypoxic-ischemic neuronal injury in all areas sampled. There was no evidence of mesial temporal sclerosis, gliosis, neuronal loss, or cortical dysplasia. No histologic findings that have been associated with epilepsy were found.

Interestingly, and as a possible forewarning, when the child's mother was a child, she had a febrile seizure that led to respiratory arrest, thereby requiring cardiorespiratory resuscitation and a weeklong hospitalization.

\section{DISCUSSION}

Febrile seizures are usually considered relatively benign, occur most commonly between the ages of 6 months and 6 years, have a peak incidence at 18 months, and occur in $2-5 \%$ of children before the age of 5 years $(1,2)$. In the case presented here, a 2.75-year-old child with a history of complex febrile GTCSs had a suspected seizure in bed at night. She was found unresponsive, pulseless, and apneic approximately $30 \mathrm{~min}$ after she was last checked by her father. Although a seizure was not witnessed, it was suspected based on the circumstances, clinical findings surrounding death, and aspects of the clinical history. First, the child had a history of multiple GTCSs during febrile episodes and had two febrile GTCSs on the same day she was later found unresponsive in bed. Second, the child was found with her face turned slightly downward, near her sister's shoulder and the pillow, a position that may have limited airflow, a common finding during seizures that lead to death. Remarkably, the scenario surrounding this death is commonly found in epilepsy patients who die from SUDEP after 
a GTCS. However, this child did not have a diagnosis of epilepsy and therefore, by definition, cannot be called SUDEP.

Despite semantic and classification differences, the commonalities between this case and the majority of SUDEP (Table 1) cases suggest a common pathophysiologic mechanism. The MORTEMUS study (8) revealed that SUDEP cases are often induced by a GTCS while the individual is in bed, at night, often during sleep and facedown. A GTCS can lead to activation/ inactivation of brain regions that are functionally connected to the brainstem respiratory network (4), which can result in ictal and postictal respiratory dysfunction and oxygen desaturation. Recent work by Dlouhy et al. (10) found that seizure spread to the amygdala as well as electrical stimulation of the amygdala resulted in apnea and oxygen desaturation in humans. Being prone, facedown in bed, and asleep would likely exacerbate this oxygen desaturation. This hypoxemia may ultimately lead to bradycardia, asystole, and death. The fact that many SUDEP cases are found prone in bed suggests that this position plays a role in the pathophysiology. The prone position where the face may be covered by pillows or blankets likely predisposes to impaired ventilation and oxygenation during inhibition of respiratory motor output. This position also suggests a loss of arousal by patients, as they do not sense the alarm of rising $\mathrm{CO}_{2}$ concentrations (4). Mechanistically, this loss of arousal during the peri-ictal period may be explained by an especially striking observation in the study by Dlouhy et al. (10) in which apnea evoked by amygdala stimulation was not accompanied by dyspnea. In fact, the patients were completely unaware that they had stopped breathing. Therefore, a loss of arousal to $\mathrm{CO}_{2}$ may occur with amygdala seizures.

The child discussed here was known to have apnea (witnessed and described by the patient's mother who is an experienced health-care provider) during her febrile seizures with oxygen desaturation causing cyanosis, further supporting the hypothesis that a seizure in this case may have led to a cascade of events similar to what is seen in SUDEP cases. Strikingly, and supporting the likelihood that a seizure led to death in this case, the mother of the child also experienced a febrile seizure in childhood, which led to respiratory arrest requiring cardiorespiratory resuscitation and prolonged hospitalization.

TABLE 1 | Shared and different characteristics between SUDC, SUDEP, and case presented here.

\begin{tabular}{lccc}
\hline Clinical feature & SUDC & SUDEP & Case \\
\hline Occurrence at night & Yes & Yes & Yes \\
Occurrence during sleep & Yes & $+/-$ & Yes \\
Prone positioning & Yes & Yes & Yes \\
Facedown & Yes & Yes & Yes \\
History of febrile seizures & Yes & $+/-$ & Yes \\
History of GTCS & Yes & Yes & Yes \\
Suspected respiratory dysfunction & Unknown & Yes & Suspected \\
at death & & & \\
History of apneic seizures & Unknown & Unknown & Yes \\
Apparent lack of struggle at death & Yes & Yes & Yes \\
History of epilepsy & No & Yes & No \\
Age at death & 1-5 years & Any age & 2.75 years \\
\hline
\end{tabular}

SUDC, sudden unexplained death in childhood; SUDEP, sudden unexpected death in epilepsy; GTCS, generalized tonic-clonic seizure.
Increasing evidence, as discussed above, suggests peri-ictal respiratory dysfunction as the primary cause for SUDEP. However, death still may have occurred through other mechanisms in this child. Although evidence suggests a seizure occurred immediately prior to death, it is possible that a seizure immediately proceeding death did not occur. Prolonged autonomic effects from the two complex febrile seizures occurring earlier in the day could have resulted in cardiac changes or arrhythmia at night resulting in death.

We acknowledge that this is a rare case. However, in a large population-based cohort of children, Vestergaard et al. (11) found that mortality is increased in children during the 2 years after having a complex febrile seizure. Additionally, retrospective analysis of 121 cases of SUDC as part of the San Diego SUDC research project found that $48.8 \%$ of SUDC cases had a personal and/or family history of febrile seizures and therefore was a significant risk factor for SUDC (7). In another retrospective review of 123 consecutive children with SUDC reported to the SUDC program of the SUDC Foundation, 31.7\% of SUDC cases had a personal history of febrile seizures (6). An ongoing prospective SUDC registry may help support the hypothesis that febrile seizures can lead to death with similar pathophysiology to SUDEP and how commonly this occurs.

The cause of febrile seizures is unclear and likely multifactorial. No single susceptibility gene has been found to cause febrile seizures (12). However, some genetic mutations that result in epilepsy syndromes have a component of recurrent febrile seizures (2). Mutations in SCN1A, a sodium ion channel, can result in both generalized epilepsy with febrile seizures plus (GEFS+) and Dravet syndrome (DS) $(2,12)$. The risk of SUDEP is high in children with DS, probably more than other infantile epilepsies (13). It is unclear why patients with DS have a high rate of early mortality but it may be related to the severity and frequency of the seizures (5). Mutations in SCN1B are linked to GEFS+, temporal lobe epilepsy, and DS (14-16). Further work in linking SCN1B and other genes encoding ion channels in the brain to SUDEP are needed. The child in this case did not fit the clinical picture of having an epilepsy syndrome with febrile seizures.

As further progress is made in defining the mechanisms involved in SUDEP and SUDC, more of these cases will be "explained" with respect to pathophysiologic causes. Mechanistic overlap likely exists, and further evidence may support that some cases of SUDC are due to unrecognized seizures, and some cases of sudden death are induced by provoked seizures. As that happens, these categorical terms will begin to lose meaning and hinder our ability to accurately describe the cause of death-therefore, as we learn more, it will be more rational to classify these deaths based on pathophysiologic mechanism.

\section{CONCLUSION}

This case suggests that febrile seizures can lead to sudden unexpected death in children through mechanisms similar to those involved in SUDEP. Further study is needed to better understand how seizures of any kind, including provoked and epileptic, cause death. As we learn more, it will become possible to divide SUDC into subgroups based on pathophysiologic mechanism. 


\section{ETHICS STATEMENT}

The parents of the child described here consented to this study and report.

\section{AUTHOR CONTRIBUTIONS}

Author contributions to the study and manuscript preparation include the following. Conception and design: BD, MN, and GR. Acquisition of data, and analysis and interpretation of data:

\section{REFERENCES}

1. Chungath M, Shorvon S. The mortality and morbidity of febrile seizures. Nat Clin Pract Neurol (2008) 4:610-21. doi:10.1038/ncpneuro0922

2. Bast T, Carmant L. Febrile and other occasional seizures. Handb Clin Neurol (2013) 111:477-91. doi:10.1016/B978-0-444-52891-9.00052-X

3. Nashef L, So EL, Ryvlin P, Tomson T. Unifying the definitions of sudden unexpected death in epilepsy. Epilepsia (2012) 53:227-33. doi:10.1111/j.15281167.2011.03358.x

4. Dlouhy BJ, Gehlbach BK, Richerson GB. Sudden unexpected death in epilepsy: basic mechanisms and clinical implications for prevention. J Neurol Neurosurg Psychiatry (2016) 87:402-13. doi:10.1136/jnnp-2013307442

5. Devinsky O, Hesdorffer DC, Thurman DJ, Lhatoo S, Richerson G. Sudden unexpected death in epilepsy: epidemiology, mechanisms, and prevention. Lancet Neurol (2016) 15:1075-88. doi:10.1016/S1474-4422(16) 30158-2

6. Hesdorffer DC, Crandall LA, Friedman D, Devinsky O. Sudden unexplained death in childhood: a comparison of cases with and without a febrile seizure history. Epilepsia (2015) 56:1294-300. doi:10.1111/epi.13066

7. Hefti MM, Kinney HC, Cryan JB, Haas EA, Chadwick AE, Crandall LA, et al. Sudden unexpected death in early childhood: general observations in a series of 151 cases: part 1 of the investigations of the San Diego SUDC research project. Forensic Sci Med Pathol (2016) 12:4-13. doi:10.1007/ s12024-015-9731-3

8. Ryvlin P, Nashef L, Lhatoo SD, Bateman LM, Bird J, Bleasel A, et al. Incidence and mechanisms of cardiorespiratory arrests in epilepsy monitoring units (MORTEMUS): a retrospective study. Lancet Neurol (2013) 12:966-77. doi:10.1016/S1474-4422(13)70214-X

9. Massey CA, Sowers LP, Dlouhy BJ, Richerson GB. Mechanisms of sudden unexpected death in epilepsy: the pathway to prevention. Nat Rev Neurol (2014) 10:271-82. doi:10.1038/nrneurol.2014.64

10. Dlouhy BJ, Gehlbach BK, Kreple CJ, Kawasaki H, Oya H, Buzza C, et al. Breathing inhibited when seizures spread to the amygdala and all the authors. Draft of the article: BD. Critical revision of the article and review of submitted version of the manuscript: all the authors. Approval of the final version of the manuscript on behalf of all the authors, administrative/technical/material support, and study supervision: BD.

\section{FUNDING}

The lead author, BD, was funded by the NINDS/CNS Getch Scholar Career Development Award.

upon amygdala stimulation. J Neurosci (2015) 35:10281-9. doi:10.1523/ JNEUROSCI.0888-15.2015

11. Vestergaard M, Pedersen MG, Ostergaard JR, Pedersen CB, Olsen J, Christensen J. Death in children with febrile seizures: a population-based cohort study. Lancet (2008) 372:457-63. doi:10.1016/S0140-6736(08)61198-8

12. Camfield P, Camfield C. Febrile seizures and genetic epilepsy with febrile seizures plus (GEFS+). Epileptic Disord (2015) 17:124-33. doi:10.1684/ epd.2015.0737

13. Kalume F. Sudden unexpected death in Dravet syndrome: respiratory and other physiological dysfunctions. Respir Physiol Neurobiol (2013) 189:324-8. doi:10.1016/j.resp.2013.06.026

14. Wallace RH, Wang DW, Singh R, Scheffer IE, George AL Jr, Phillips HA, et al. Febrile seizures and generalized epilepsy associated with a mutation in the Na+-channel beta1 subunit gene SCN1B. Nat Genet (1998) 19:366-70. doi:10.1038/1252

15. Scheffer IE, Harkin LA, Grinton BE, Dibbens LM, Turner SJ, Zielinski MA, et al. Temporal lobe epilepsy and GEFS+ phenotypes associated with SCN1B mutations. Brain (2007) 130:100-9. doi:10.1093/brain/awl272

16. Ogiwara I, Nakayama T, Yamagata T, Ohtani H, Mazaki E, Tsuchiya S, et al. A homozygous mutation of voltage-gated sodium channel beta(I) gene SCN1B in a patient with Dravet syndrome. Epilepsia (2012) 53:e200-3. doi:10.1111/ epi. 12040

Conflict of Interest Statement: The authors report no conflict of interest concerning the materials or methods used in this study or the findings specified in this paper.

Copyright (C) 2017 Dlouhy, Ciliberto, Cifra, Kirby, Shrock, Nashelsky and Richerson. This is an open-access article distributed under the terms of the Creative Commons Attribution License (CC BY). The use, distribution or reproduction in other forums is permitted, provided the original author(s) or licensor are credited and that the original publication in this journal is cited, in accordance with accepted academic practice. No use, distribution or reproduction is permitted which does not comply with these terms. 